\title{
Ovarian Transposition Strategy in Patients with Cervical Cancer Who Undergo Pelvic Radiation: Proposal of Ovarian Placement Based on Virtual Simulations
}

\author{
Authors: \\ *Gabriel Oliveira Bernardes Gil,1,2 Cassiano Asano,' Maria Luísa Braga \\ Vieira Gil, ${ }^{1}$ Warne Andrade, ${ }^{2}$ Eduardo Batista Cândido, 2,3 Marcos \\ Regalin, ${ }^{1}$ Izabella Nobre Queiroz, ${ }^{4}$ Darly Gomes Soares Delfino, ${ }^{5}$ Farley \\ Soares Cantídio, ${ }^{4}$ Agnaldo Lopes Silva-Filho, ${ }^{2,3}$ \\ 1. Mater Dei Hospital, Belo Horizonte, Brazil \\ 2. Botucatu Medical School, São Paulo State University, Botucatu, Brazil \\ 3. Federal University of Minas Gerais, Belo Horizonte, Brazil \\ 4. Baleia Hospital, Belo Horizonte, Brazil \\ 5. Faminas-BH, Belo Horizonte, Brazil \\ *Correspondence to gabrielbgil@gmail.com
}

Disclosure: The authors have declared no conflicts of interest.

Received: $\quad 06.07 .20$

Accepted: $\quad 16.07 .21$

Keywords: Cervical cancer, ovarian failure, ovarian transposition, radiotherapy.

Citation:

EMJ Innov. 2021; DOI/10.33590/emjinnov/20-00170.

\begin{abstract}
Objective: To establish a proposal for the location for ovarian transposition, considering different irradiation techniques and time to ovarian failure.

Methods: Patients with cervical cancer in childbearing age submitted to adjuvant radiotherapy were selected. Delineation of simulated positions of the ovaries and pelvic radiation planning was done in CT, with three techniques: 3D conformal radiotherapy, intensity-modulated radiotherapy, and volumetric modulated arc radiotherapy. In order to correlate the ovaries maximal doses with the time to ovarian failure, the authors have used the one adaptation of Wallace model that predicts oocytes survival rates after radiation exposure.
\end{abstract}

Results: Thirteen patients who were being treated between 2008 and 2017 were studied. When the ovaries were positioned $10 \mathrm{~cm}$ cranially from the sacral promontory, the pelvic radiation entails a decrease of $20 \%$ in the time to ovarian failure compared with that expected for a female at the same age without irradiation exposition. The placement of the ovaries $<5 \mathrm{~cm}$ cranially from the sacral promontory results in a decrease $>90 \%$. There was no difference in time to ovarian failure between the radiation treatment techniques tested: 3D conformal radiotherapy, intensity-modulated radiotherapy, and volumetric modulated arc radiotherapy $(p=0.197)$.

Conclusions: The present study, based on virtual simulations, is the first to use the sacral promontory as a reference for a proposal of ovarian location with transposition. The authors have correlated the position of the ovaries and percentage of decrease in time to ovarian failure. These findings can potentially improve the management and counselling of patients with cervical cancer in childbearing age and deserve clinical validation. 


\section{INTRODUCTION}

Cervical cancer is a public health problem worldwide, with an annual incidence of 528,000 new cases and 226,000 deaths. ${ }^{1}$ The distribution of the disease is bimodal, with a peak at 35-39 years old and a second peak at 75-79 years old. ${ }^{2}$ It is estimated that $>30 \%$ of cervical cancers are diagnosed in females in their reproductive age. ${ }^{3}$ Treatment of cervical cancer is based on surgery, radiotherapy, and chemotherapy. The best therapeutic choice must consider the disease's staging, age, clinical condition, available local resources, and the patient's desire for family planning and preservation of fertility.

Despite playing a key role in the management of cervical cancer, radiation can induce premature ovarian failure. Radiotherapy is now a well-known cause of ovarian damage, leading earlier menopause with permanent infertility. Doses $>6$ Gy in total body irradiation in young people induce premature ovarian failure, whereas prepubertal individuals can tolerate even higher radiation doses. ${ }^{4}$ Wallace et al. demonstrated that the dose necessary to destroy $50 \%$ of the primordial follicles is less than $2 \mathrm{~Gy}^{5}$ and that 4 Gy can produce infertility in one-third of young females and in almost all females over the age of 40 years. ${ }^{6}$ The degree of ovarian impairment is related to the volume treated, total irradiation dose, fractionation schedule, and age at the time of treatment, with older people being at greater risk of damage. ${ }^{7}$

In addition to issues related to the reproductive future arising from the ovarian failure, premature menopause is associated with cardiovascular disease, osteoporosis, genital atrophy, vasomotor symptoms, and a significant impact on quality of life. ${ }^{8}$ The term 'induced menopause' has been defined by the North American Menopause Society (NAMS) as the cessation of menstruation following bilateral oophorectomy or iatrogenic ablation of ovarian function resulting from delivery of chemotherapy or pelvic radiation. ${ }^{9}$ The onset of symptoms can occur within 12 weeks of initiation of pelvic radiation therapy. ${ }^{10}$ Management of climacteric symptoms is critical in efforts to optimise the quality of life. However, potential hormone stimulation has raised concern over the safety of hormone therapy in this population. ${ }^{11-13}$
In order to minimise the effects of induced menopause, ovarian transposition or oophoropexy can be surgically performed to remove the ovaries from the area to be irradiated. This procedure may prevent induced menopause and ovaries may be used at a later date for oocyte retrieval, in vitro fertilisation, and achieving pregnancy through surrogacy if appropriate. ${ }^{14}$ Ovarian transposition is also described in the context of paediatric tumours and some pelvic neoplasms in young women for the purpose of ovarian and even fertility preservation. ${ }^{15,16}$ This procedure can be performed by laparotomy or laparoscopy, it has low morbidity, is safe from the oncological point of view, and, in the context of patients with cervical cancer, may be indicated for young patients with indication of radiation. ${ }^{17-20}$

Techniques have been described to relocate the ovaries to the paracolic gutters, behind the uterus, or to anterolateral positions above the umbilicus. ${ }^{21}$ There is no consensus as to where is the best position in which the ovaries should be implanted.16,22-26 Therefore, the aim of this study was to establish a proposal, based on virtual simulations, to suggest the location of the ovaries in the transposition, considering different radiation techniques and time to ovarian failure.

\section{MATERIALS AND METHODS}

The patients evaluated included females diagnosed with cervical cancer in childbearing age, defined as age under 51 years, without climacteric symptoms, submitted to adjuvant pelvic radiotherapy, where all treatments did not use any type of ovarian preservation such as ovarian transposition. Electronic medical records were used to collect their pathological staging, histological type, patient's age, weight, height, and $\mathrm{BMI}$ at time of diagnosis. The present study was approved by the ethics committee of the involved institution. According to the criteria above, the authors retrospectively identified 13 patients treated between January 2008 and July 2017.

The CT obtained was used for the virtual simulation of the transposed ovaries and for radiotherapy planning. Delineation of the positions of 54 of the simulated ovaries was performed by two radiation oncologists and two experienced oncology gynaecologists. Radiotherapy planning was performed in three 
different techniques: 3D conformal radiotherapy, intensity-modulated radiation therapy (IMRT), and volumetric modulated arc therapy (VMAT), all using beams of $10 \mathrm{MV}$ with dose prescription of 45 Gy with $95 \%$ of coverage in target volume. The target volume used for radiotherapy planning was planning target volume, which consisted of the clinical target volume (CTV) plus a $5 \mathrm{~mm}$ margin. The CTV contouring included the common, external, internal iliac, and presacral lymph node region. The upper $3 \mathrm{~cm}$ of the vagina and paravaginal soft tissue lateral to the vagina were also included. The superior border of the CTV began 5-7 $\mathrm{mm}$ below the L4-L5 interspace, such as would customarily be used in a conventional four-field box. ${ }^{27}$

Intending to associate the dose received by the ovaries with its function, the survival equation obtained by Wallace et al. ${ }^{28}$ was used. The theoretical decrease in time to ovarian failure in percentage was calculated as a ratio between the time to ovarian failure after a specified radiation dose and time to ovarian failure with no radiation exposure. The algorithm was applied for the maximum dose values in each outlined structure that simulated the ovarian position. The distance between the sacral promontory and the inferior border of the simulated ovaries in the cranialcaudal axis was associated with time to ovarian failure. The authors also evaluated the impact of mediolateral and anteroposterior displacement in time to ovarian failure.

\section{STATISTICAL ANALYSIS}

For the statistical analysis, $\mathrm{R}$ software (version 3.4.2) was used. The effect of the distance between the sacral promontory and the inferior border of the simulated ovaries in the cranial-caudal axis on premature ovarian failure was studied with three non-linear regression models: Log-Logistic, Weibull, and Log-Normal. In order to select the best model, the Akaike information criterion was used. ${ }^{29,30}$ According to this method, the Weibull model was selected. To verify if the relationship between the decrease in time to ovarian failure and distance from sacral promontory and the inferior border of the simulated ovaries in the cranial-caudal axis was different between the radiation techniques, heights, weights, and BMls, analysis of variance, often referred to as ANOVA, was used. ${ }^{31}$ Then, models were adjusted for each of the classes of interest that were obtained through the median of the variables. In all models, the fit quality was verified by the 'lack-of-fit' test. ${ }^{32}$

\section{RESULTS}

The majority of the patients had squamous cell carcinoma, Stage IB or IIA. Regarding the characterisation variables, these are presented as mean \pm standard deviation. The mean age of the patients was $36.4 \pm 8.6$ years, the mean height was $1.61 \pm 0.05 \mathrm{~m}$, the mean weight was $69 \pm 20 \mathrm{~kg}$, and the mean BMl was $26.8 \pm 8.3 \mathrm{~kg} / \mathrm{m}^{2}$, with the minimum of $20.8 \mathrm{~kg} / \mathrm{m}^{2}$ and the maximum of $46.7 \mathrm{~kg} / \mathrm{m}^{2}$.

Different positions of the simulated ovaries in the mediolateral and anteroposterior axis did not show any difference in time to ovarian failure. Figure 1 shows the relationship between the decrease in time to ovarian failure and distance from sacral promontory to the inferior border of the simulated ovaries in the cranial-caudal axis. It is possible to verify that ovaries positioned $10 \mathrm{~cm}$ cranially from the sacral promontory result in a decrease of $20 \%$ in the time to ovarian failure compared with what would be expected for a woman at the same age without radiation exposure. The placement of the ovaries $<5 \mathrm{~cm}$ cranially from the sacral promontory resulted in a decrease $>90 \%$ in the time to ovarian failure. There was no difference in ovarian preservation between the 3D conformal radiotherapy, IMRT, and VMAT techniques $(p=0.197)$.

Table 1 illustrates the decrease in the time to ovarian failure after specified radiation dose received by the ovaries compared with non-irradiated females of the same age associated to the different positions of the ovaries cranially from the sacral promontory. According to the 'lack-of-fit' test, the model presented a good fit $(p=1.000)$. For simulated ovaries positioned $7 \mathrm{~cm}$ above the promontory in the caudal-cranial axis, the mean dose was 6.7 Gy or smaller and the decrease in time to ovarian failure would be $<50 \%$, whereas for ovaries positioned $10 \mathrm{~cm}$ above the promontory the mean dose would be 1.4 Gy or smaller and the decrease in time to ovarian failure would be $<20 \%$ (Figure 2 ). These findings were consistent regardless of the patient's height, weight, and BMI. 


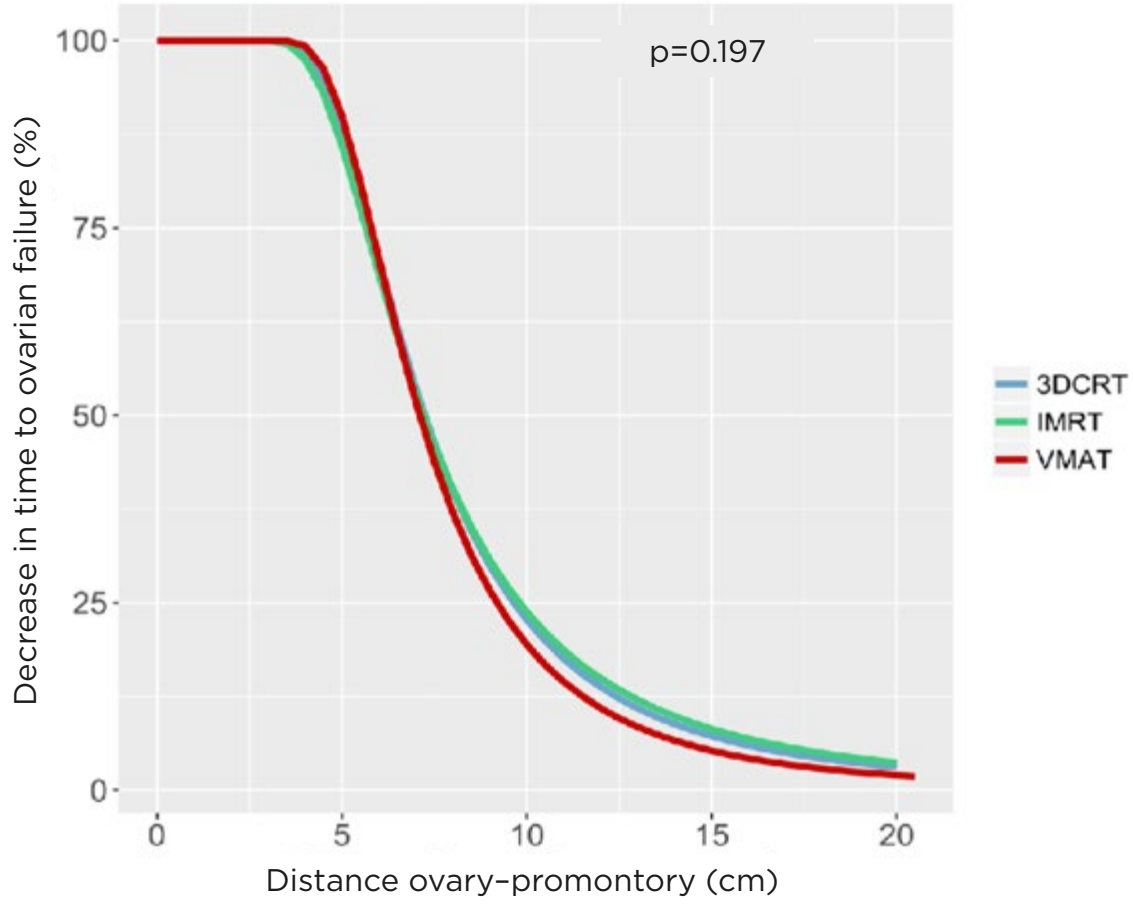

Figure 1: The relationship between the decrease in time to ovarian failure and the distance from sacral promontory in cranio-caudal axis to inferior border of the ovaries.

IMRT: intensity-modulated radiotherapy; VMAT: volumetric modulated arc radiotherapy; 3DCRT: 3D conformal radiotherapy.

Table 1: Decrease in the time to ovarian failure compared with non-irradiated females associated to the different positions of the ovaries cranially from the sacral promontory.

\begin{tabular}{|l|l|}
\hline $\begin{array}{l}\text { Decrease in the time } \\
\text { to ovarian failure (\%) }\end{array}$ & $\begin{array}{l}\text { Cranial distance from the sacral } \\
\text { promontory }(95 \% \mathrm{Cl})(\mathrm{cm})\end{array}$ \\
\hline 90 & $4.9(4.7-5.0)$ \\
\hline 80 & $5.5(5.3-5.6)$ \\
\hline 70 & $6.0(5.9-6.1)$ \\
\hline 60 & $6.6(6.5-6.7)$ \\
\hline 50 & $7.2(7.1-7.3)$ \\
\hline 40 & $7.9(7.8-8.1)$ \\
\hline 30 & $8.9(8.7-9.1)$ \\
\hline 20 & $10.4(10.1-10.7)$ \\
\hline 10 & $13.3(12.7-13.8)$ \\
\hline
\end{tabular}

Decrease in time to ovarian failure as a function of distance from the sacral promontory to the inferior border of the ovary in the cranio-caudal axis.

Cl: confidence interval. 


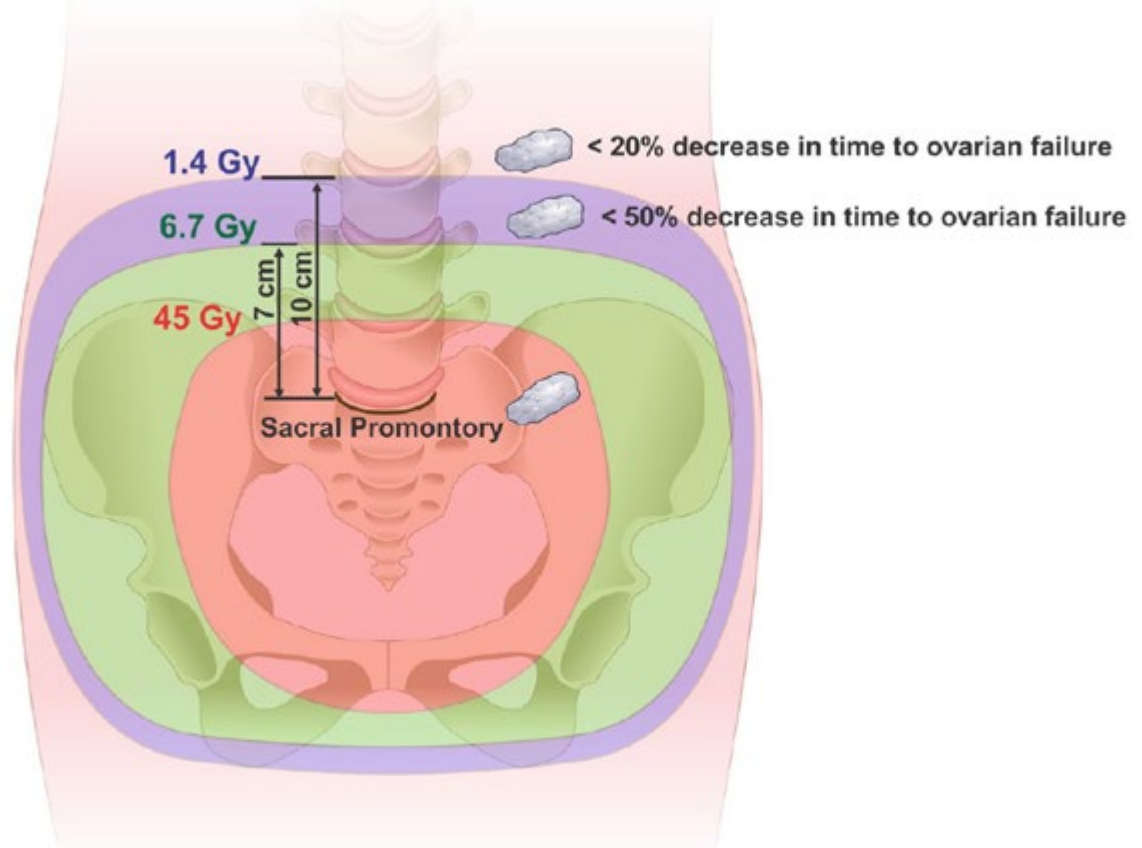

Figure 2: Schematic representation of the simulated positions of the ovaries and percentage of decrease in time to ovarian failure.

The radiation isodoses are illustrated by a classic planning of pelvic radiotherapy for cervical cancer. In it, the isodose of $45.0 \mathrm{~Gy}, 6.7 \mathrm{~Gy}$, and 1.4 Gy are observed and its position in relation to the promontory. Ovaries are represented by simulating the transposition height.

\section{DISCUSSION}

Transposition of the ovaries out of the pelvic irradiation field has long been used for this purpose. ${ }^{33}$ The general agreement appears to be as high and as lateral as possible from the original sites to be away from the pelvic radiotherapy field; however, there is no consensus concerning where to transpose ovaries. In this regard, the authors used virtual simulations to propose a practical location for the ovarian transposition in an attempt to preserve ovarian function regarding hormonal production and also fertility preservation. ${ }^{34}$ Models are used because, in some way, they are more accessible and convenient. A model is a representation of some reality that embodies some essential and interesting aspects of that reality, but not all of it. ${ }^{35}$

Using a standard pelvic radiation for cervical cancer with the upper limit of the radiation fields located at intervertebral space between $L 4$ and L5, the authors could observe that $7 \mathrm{~cm}$ above

the sacral promontory in the caudal-cranial axis the decrease in time to ovarian failure was $50 \%$ or less. Whereas for ovaries positioned 10 $\mathrm{cm}$ above the sacral promontory, the decrease in time to ovarian failure would be $<20 \%$. The authors' findings are generally consistent with other studies that recommend a cranial location to ovarian transposition evaluating clinical outcomes. One study suggested an approach to transpose ovaries to a high anterolateral position at least 3-4 cm above the umbilical line and reported good results for those under 40 years old. ${ }^{36}$ In a retrospective analysis of 53 cases, two surgical clips were applied to the upper and lower borders of each transposed ovary so that the position of the transposed ovaries could be identified. They have shown better preservation rates were obtained when the ovaries were implanted $1.5 \mathrm{~cm}$ above the iliac crest. ${ }^{25}$

Unlike that observed in the craniocaudal axis, the mediolateral or anteroposterior displacement of the simulated ovaries did not show any 
difference in time to ovarian failure. One possible explanation to be considered is that the classical pelvic radiation's fields are co-planar and produce a low dose distribution that varies a little in the mediolateral and anteroposterior axis. Usually, the studies evaluating the ovarian transposition use as anatomical reference structures to be considered for radiotherapy treatment planning. The authors' study was the first to use the sacral promontory as a reference to ovarian transposition, with the potential advantage as a structure that can be easily identified during the surgical procedure. The sacral promontory is also useful in treatment planning for radiation therapy.

The present study was based on virtual simulations and did not consider any other clinical outcome. Published data confirm and generalise the concept that ovarian transposition is associated with a high preservation of ovarian function, an acceptable rate of ovarian cysts, and a low risk of metastases in the transposed ovaries. ${ }^{37}$ The authors did not consider unilateral ovarian transposition and the results could only be applied to both ovaries located at the same distance from the sacral promontory in the cranial-caudal axis. Another important and not considered issue is the influence of the absence of the uterus in the ovarian function. It is unresolved whether it is the surgery itself or the underlying condition leading to hysterectomy that is the cause of earlier ovarian failure. ${ }^{38}$ The results are only applicable in the scenarios of adjuvant pelvic radiotherapy and do not contemplate brachytherapy, para-aortic irradiation, or even the chemotherapy impact on oocytes damage.
The authors' study was not able to demonstrate a difference in time to ovarian failure with the three radiation techniques studied. The authors attribute that to the fact that they did not modulate the field to avoid the ovaries. Sophisticated external beam irradiation techniques, such as IMRT and VMAT, could offer by means of 'dose painting' a considerable reduction in dose to the transposed ovaries. After ovarian transposition using surgical clips, the ovaries could be identified in the planning CT and an avoidance volume created by the radiation oncologist. This can guarantee that even a lower dose of radiation will be delivered to that volume. ${ }^{39}$

\section{CONCLUSION}

In summary, this study was an attempt based on virtual simulations to define the location of the ovaries in the ovarian transposition. The authors used the sacral promontory as the anatomical landmark for ovarian placement, which is accessible by the surgeons and radiation oncologists. A cranial distance $\geq 10 \mathrm{~cm}$ from the sacral promontory has shown a minimal decrease in time to ovarian failure. The proposed model seems to be easy to apply in clinical practice as well as to provide information for medical decision-making. These findings can potentially improve the management and counselling of patients with cervical cancer in childbearing age. More studies with clinical outcomes and follow-up of the patients are needed to validate and optimise the model proposed.

\section{References}

1. Torre LA et al. Global cancer statistics, 2012. CA Cancer J Clin. 2015;65(2):87-108.

2. Quinn $M$ et al. Effect of screening on incidence of and mortality from cancer of cervix in England: evaluation based on routinely collected statistics. BMJ. 1999;318(7188):904-8.

3. Nguyen C et al. Management of stage I cervical cancer in pregnancy. Obstet Gynecol Surv. 2000;55(10):633-43.

4. Rodriguez-Wallberg KA, Oktay K. Options on fertility preservation in female cancer patients. Cancer Treat Rev. 2012;38(5):354-61.
5. Wallace WHB et al. Ovarian failure following abdominal irradiation in childhood: natural history and prognosis. Clin Oncol (R Coll Radiol). 1989;1(2):75-9.

6. Wallace WHB et al. The radiosensitivity of the human oocyte. Hum Reprod. 2003;18(1):117-21.

7. Adriaens I et al. The current knowledge on radiosensitivity of ovarian follicle development stages. Hum Reprod Update. 2009;15(3):35977.

8. Plante M, Roy M. Fertility-preserving options for cervical cancer. Oncology (Williston Park). 2006;20(5):479-88.
9. Shifren JL, Gass MLS; NAMS Recommendations for Clinical Care of Midlife Women Working Group. The North American Menopause Society recommendations for clinical care of midlife women. Menopause. 2014;21(10):1038-62.

10. Hinds L, Price J. Menopause, hormone replacement and gynaecological cancers. Menopause Int. 2010;16(2):89-93.

11. Michaelson-Cohen R, Beller U. Managing menopausal symptoms after gynecological cancer. Curr Opin Oncol. 2009;21(5):407-11.

12. Rossouw JE et al.; Writing Group for the Women's Health Initiative 
Investigators. Risks and benefits of estrogen plus progestin in healthy postmenopausal women: principal results from the Women's Health Initiative randomized controlled trial. JAMA. 2002;288(3):321-33.

13. Grady D et al.; HERS Research Group. Cardiovascular disease outcomes during 6.8 years of hormone therapy: Heart and Estrogen/progestin Replacement Study follow-up (HERS II). JAMA. 2002;288(1):49-57.

14. Koliopoulos $\mathrm{G}$ et al. Conservative surgical methods for FIGO stage IA2 squamous cervical carcinoma and their role in preserving women's fertility. Gynecol Oncol. 2004:93(2):469-73.

15. Lester-Coll $\mathrm{NH}$ et al. Preserving fertility in adolescent girls and young women requiring craniospinal irradiation: a case report and discussion of options to be considered prior to treatment. $\mathrm{J}$ Adolesc Young Adult Oncol. 2014;3(2):96-9.

16. Nahhas WA et al. Lateral ovarian transposition. Ovarian relocation in patients with Hodgkin's disease. Obstet Gynecol. 1971;38(5):785-8.

17. Arian SE et al. Ovarian transposition: a surgical option for fertility preservation. Fertil Steril. 2017;107(4):e15.

18. Yamamoto R et al. A study of risk factors for ovarian metastases in stage IB-IIIB cervical carcinoma and analysis of ovarian function after a transposition. Gynecol Oncol. 2001;82(2):312-6.

19. McCall ML et al. Conservation of ovarian tissue in the treatment of carcinoma of the cervix with radical surgery. Am J Obstet Gynecol. 1958;75(3):590-600.

20. Pahisa J et al. Laparoscopic ovarian transposition in patients with early cervical cancer. Int J Gynecol Cancer. 2008;18(3):584-9

21. Clough $\mathrm{KB}$ et al. Laparoscopic unilateral ovarian transposition prior to irradiation: prospective study of 20 cases. Cancer. 1996;77(12):2638-45.

22. Fujiwara K et al. Subcutaneous transposition of the ovary following hysterectomy. Int J Gynaecol Obstet. 1997;58(2):223-8.

23. Gareer W et al. Needle oophoropexy: a new simple technique for ovarian transposition prior to pelvic irradiation. Surg Endosc. 2011;25(7):2241-6.

24. Hodel $\mathrm{K}$ et al. The role of ovarian transposition in conservation of ovarian function in radical hysterectomy followed by pelvic radiation. Gynecol Oncol. 1982;13(2):195-202

25. Hwang $\mathrm{JH}$ et al. Association between the location of transposed ovary and ovarian function in patients with uterine cervical cancer treated with (postoperative or primary) pelvic radiotherapy. Fertil Steril. 2012;97(6):1387-93.

26. Moawad NS et al. Laparoscopic ovarian transposition before pelvic cancer treatment: ovarian function and fertility preservation. J Minim Invasive Gynecol. 2017;24(1):28-35.

27. Small $W$ et al. Consensus guidelines for delineation of clinical target volume for intensity-modulated pelvic radiotherapy in postoperative treatment of endometrial and cervical cancer. Int J Radiat Oncol Biol Phys. 2008;71(2):428-34.

28. Wallace WHB et al. Predicting age of ovarian failure after radiation to a field that includes the ovaries. Int J Radiat Oncol Biol Phys. 2005;62(3):738-44.

29. Finney DJ, Statistics for Biologists
(1980) $4^{\text {th }}$ edition, London, New York: Chapman \& Hall, pp.196.

30. Seber GAF, Wild CJ, "Statistical inference," Nonlinear Regression (2005), New Jersey: John Wiley \& Sons, Inc., pp.191-269.

31. Bewick $V$ et al. Statistics review 9: one-way analysis of variance. Crit Care. 2004;8(2):130-6.

32. Bates DM, Watts DG, Nonlinear Regression Analysis and Its Applications (1988), Hoboken: John Wiley \& Sons, Inc.

33. Anderson B et al. Ovarian transposition in cervical cancer. Gynecol Oncol. 1993;49(2):206-14

34. Chan JL, Wang ET. Oncofertility for women with gynecologic malignancies. Gynecol Oncol. 2016;144(3):631-6.

35. Pérez-Andújar A et al. The predicted relative risk of premature ovarian failure for three radiotherapy modalities in a girl receiving craniospinal irradiation. Phys Med Biol. 2013:58(10):3107-23.

36. Huang KG et al. A new approach for laparoscopic ovarian transposition before pelvic irradiation. Gynecol Oncol. 2007;105(1):234-7.

37. Gubbala K et al. Outcomes of ovarian transposition in gynaecological cancers; a systematic review and meta-analysis. J Ovarian Res. 2014;7(1):69.

38. Moorman PG et al. Effect of hysterectomy with ovarian preservation on ovarian function. Obstet Gynecol. 2011;118(6):127-9

39. Ghadjar P et al. Modern radiation therapy and potential fertility preservation strategies in patients with cervical cancer undergoing chemoradiation. Radiat Oncol. 2015;10:50. 